\title{
A Pharmacokinetic Analysis of a Novel Fixed Dose Oral Combination of Paracetamol and Ibuprofen, with Emphasis on Food Effect
}

\section{Hartley C Atkinson ${ }^{1}$, Ioana Stanescu', Charles PH Beasley1, Isam I Salem ${ }^{2}$ and Chris Frampton ${ }^{3 *}$}

${ }^{1}$ AFT Pharmaceuticals Ltd, P O Box 33-203, Takapuna, Auckland 0740, New Zealand

${ }^{2}$ International Pharmaceutical Research Centre, 1 Queen Rania Street - Sport City Circle, Amman 11196, Jordan

${ }^{3}$ University of Otago, P O Box 4345, Christchurch 8140, New Zealand

\begin{abstract}
Purpose: The published literature asserts that the individual pharmacokinetic parameters of ibuprofen and paracetamol are not altered following concurrent administration in a fasted state. The present study was performed to confirm these observations for a novel fixed dose oral combination (Maxigesic ${ }^{\circledR}$ ) containing paracetamol 500 $\mathrm{mg}$ and ibuprofen $150 \mathrm{mg} / \mathrm{tablet}$. Additionally, the effect of food on the pharmacokinetic profile of the Maxigesic ${ }^{\circledR}$
\end{abstract} formulation was assessed.

Methods: A single-dose, open-label, randomized, four-way crossover pharmacokinetic study was undertaken in 28 healthy volunteers. Serial plasma samples were assayed for both paracetamol and ibuprofen concentrations using validated LC-MS/MS methods. Ratios of $C_{\max }, A \cup C_{0 \rightarrow t}$ and $A \cup C_{0 \rightarrow \infty}$ were analysed for bioequivalence as determined by $90 \%$ confidence intervals $(\mathrm{Cl})$ and $t_{\max }$ values were compared using the Wilcoxon matched pairs test.

Results: In the fasted state, pharmacokinetic parameters for ibuprofen and paracetamol were similar between the fixed dose combination and its mono-components. Ratios of $C_{\max }, A_{U C} C_{0 \rightarrow 12 h}$, and $A U C_{0 \rightarrow \infty}$ values fell within the $80-125 \%$ acceptable bioequivalence range and $t_{\max }$ values were not altered significantly.

In the fed state compared with the fasted state, the $t_{\max }$ from the fixed dose combination was significantly prolonged for paracetamol (53 vs 30 minutes) and slightly delayed for ibuprofen (53 vs 90 minutes). Slower absorption of paracetamol resulted in a reduced $\mathrm{C}_{\max }$ which was outside the $80-125 \%$ bioequivalence range. Additionally, in the fed state, the extent of absorption of both paracetamol and ibuprofen from the fixed dose combination was slightly less compared with the fasted state, although the $90 \% \mathrm{Cl}$ for the $\mathrm{AUC}_{0 \rightarrow 12 \mathrm{~h}}$ and $\mathrm{AUC} 0_{\rightarrow \infty}$ ratios were within the $80-125 \%$ bioequivalence range.

Conclusions: The concomitant administration of ibuprofen and paracetamol in a fixed dose combination $\left(\right.$ Maxigesic $^{\circledR}$ ) does not alter the pharmacokinetic profiles of either drug in the fasted state and there was no effect of food on the absorption from the fixed dose combination.

Keywords: Paracetamol; Ibuprofen; Pharmacokinetics; Combination; Food-effect

\section{Introduction}

There are many analgesic options available to physicians, but most have limitations. Paracetamol is a widely used analgesic that has been safely used for decades but it may not provide sufficient analgesia in some clinical situations necessitating dose escalation or its combination with another drug, typically an opioid.

A novel combination treatment whereby both paracetamol and ibuprofen are combined in one single tablet at a ratio of 3.3: 1 $\left(\right.$ Maxigesic $\left.^{\circledR}\right)$ has been shown to provide superior pain relief versus its individual components [1]. Both mono-components of Maxigesic ${ }^{\mathbb{B}}$ have a long history of use and their pharmacokinetic profiles are well characterised. Previous bioequivalence studies conducted with other fixed dose combination products containing paracetamol $650 \mathrm{mg}$ and ibuprofen $400 \mathrm{mg}$ [2] and paracetamol $1000 \mathrm{mg}$ and ibuprofen $400 \mathrm{mg}$ [3] found that the addition of ibuprofen to paracetamol resulted in an increase in the rate of absorption of the latter.

The rate and extent of absorption of a variety of drugs are affected by food ingested prior to dosing [4-9] and alterations in the pharmacokinetic and pharmacodynamic profiles may have further clinical implications [10]. Reductions in plasma concentrations of both paracetamol and ibuprofen were observed following the intake of these components after a standard meal [11-18]. A single study evaluating the food-effect of a fixed dose combination of paracetamol and ibuprofen demonstrated that, like monotherapies, the absorption of both components was delayed after the intake of food resulting in significantly decreased maximum plasma concentrations. Despite these changes, the extent of absorption as measured by $\mathrm{AUC}_{0 \rightarrow t}$ and $\mathrm{AUC}_{0 \rightarrow \infty}$, was not significantly affected [3].

We hereby report the results of a single-dose, open-label, randomized, four-way cross-over pharmacokinetic study to determine and compare the pharmacokinetic parameters of $1000 \mathrm{mg}$ paracetamol and $300 \mathrm{mg}$ ibuprofen, administered orally, either alone or in combination, under fasting and in combination under fed conditions.

\section{Methods}

Trial design

*Corresponding author: Chris Frampton, University of Otago, PO Box 4345 Christchurch 8140, New Zealand, Tel: 6494880232; E-mail: Chris.frampton@otago.ac.nz

Received February 23, 2015; Accepted April 22, 2015; Published April 29, 2015

Citation: Atkinson HC, Stanescu I, Beasley CPH, Salem II, et al. (2015) A Pharmacokinetic Analysis of a Novel Fixed Dose Oral Combination of Paracetamol and Ibuprofen, with Emphasis on Food Effect. J Bioequiv Availab 7: 150-154. doi:10.4172/jbb.1000230

Copyright: @ 2015 Atkinson HC, et al. This is an open-access article distributed under the terms of the Creative Commons Attribution License, which permits unrestricted use, distribution, and reproduction in any medium, provided the original author and source are credited. 
This study was a Phase 1 , single-centre, open-label, randomized, single-dose, four-way crossover trial in 28 healthy adult participants. After an initial screening period of 14 days, there were four study periods each separated by a washout period of 7 days. The research was conducted in accordance with GCP including the Declaration of Helsinki and all applicable regulatory requirements. The trial was registered with the Australia New Zealand Clinical Trial Registry (Registration number: ACTRN 12613001151729).

\section{Participant selection}

Healthy male volunteers aged between 18 and 40 years with a Body Mass Index (BMI) of $18.5-30 \mathrm{~kg} / \mathrm{m}^{2}$ with no significant diseases as determined by medical history, physical examination and laboratory tests were recruited from the International Pharmaceutical Research Centre databases. Patients were excluded if they had taken prescription medications in the last 14 days or over the counter medications in the last four days, with the exception of the study medications. Written informed consent was obtained for each participant prior to commencing any screening procedures, which included recording demographic data, vital signs, physical examination and medical history assessments, hepatic, renal, respiratory, cardiac and gastrointestinal tests, a complete blood count and serum chemistry analysis, and concomitant medications.

\section{Treatment and study procedures}

Participants were admitted to the study site the night prior to the administration of study drugs, supervised for at least 10 hours for overnight fasting, and confined until the 12-hour blood sample was collected following study drug administration. Each participant received an oral dose of the randomly assigned treatment as outlined below:

- Treatment A: Single-Dose, 2 tablets of Paracetamol $500 \mathrm{mg}$ (total dose $1000 \mathrm{mg}$ ), fasted.

Treatment B: Single-Dose, 2 tablets of Ibuprofen $150 \mathrm{mg}$ (total dose $300 \mathrm{mg}$ ), fasted.

- Treatment C: Single-Dose, 2 tablets of MAXIGESIC ${ }^{\circledR}$ (AFT Pharmaceuticals Ltd., Auckland, NZ), (total dose paracetamol $1000 \mathrm{mg}+$ ibuprofen $300 \mathrm{mg}$ ), fasted.

- Treatment D: Single-Dose, 2 tablets of MAXIGESIC ${ }^{\circledR}$, (total dose paracetamol $1000 \mathrm{mg}$ + ibuprofen $300 \mathrm{mg}$ ), fed.

Breakfast for the fed state (Treatment D) was provided 30 minutes prior to dosing and a standard lunch and snacks were provided for all treatment groups 4 and 8 hours after study drugs were given.

Fourteen blood samples $(\sim 6 \mathrm{~mL})$ were drawn at 0.00 (one pre-dose sample), 5, 15, 30, 45 minutes and 1.00, 1.25, 1.50, 2.00, 3.00, 6.00, 8.00, 10.00 and 12.00 hours (post-dose) in each period. All blood samples were drawn from an indwelling intravenous cannula and collected in lithium heparinized tubes. After centrifugation the resulting plasma samples were immediately stored under a nominal temperature of $-70^{\circ} \mathrm{C}$ until analysed.

\section{Bioanalytical methods}

All haematology, biochemistry, urinary and serology analyses were conducted using standard methodologies within the IPRC diagnostic laboratory. Plasma concentrations of paracetamol and ibuprofen in human plasma (Li-heparin) were determined using validated Liquid
Chromatography and Mass Spectrometry procedures (LC-MS/MS).

Paracetamol plasma concentrations were analysed using API3000 and Quattro premier mass spectrometer in multiple reaction monitoring (MRM) mode using turbo Ion Spray with positive ionization. The chromatographic separation of paracetamol employed a $\mathrm{C}_{18}$ column using a mobile phase consisting of de-ionized water, formic acid and acetonitrile. Calibration curves were linear over the working range of $50-20000 \mathrm{ng} / \mathrm{mL}$ with a regression coefficient $\left(\mathrm{R}^{2}\right)$ $\geq 0.99937$. The Lower Limit of Quantification (LLOQ) was $50 \mathrm{ng} / \mathrm{mL}$ (precision 3.44\%, accuracy 93.69\%).

Ibuprofen plasma concentrations were analysed using a Sciex API 3000 and API 4000 triple quadrupole mass spectrometer in MRM mode, using turbo Ion Spray with negative ionization. The selective analysis of ibuprofen was achieved on Symmetry $\mathrm{C}_{18}$ Column by using a mobile phase consisting of ammonium formate, methanol and acetonitrile. Calibration curves were linear over the concentration rage of $50-35000 \mathrm{ng} / \mathrm{mL} \mathrm{R}^{2} \geq 0.9975$. The LLOQ was $50 \mathrm{ng} / \mathrm{mL}$ (precision $8.85 \%$, accuracy $104.25 \%)$.

\section{Pharmacokinetic analysis}

The pharmacokinetic parameters of paracetamol and ibuprofen for all subjects who completed the study were estimated using standard non-compartmental methods. The maximum plasma concentration $\left(\mathrm{C}_{\max }\right)$ and the time to peak plasma concentration $\left(\mathrm{t}_{\max }\right)$ were taken directly from the measured raw data. The area under the plasma concentration-time curve $\left(\mathrm{AUC}_{0 \rightarrow \mathrm{t}}\right)$ was calculated from measured data points from time of administration to time of last quantifiable concentration $\left(\mathrm{C}_{\mathrm{p}}\right)$ by the linear trapezoidal rule. The area under the plasma concentration-time curve extrapolated to infinity $\left(\mathrm{AUC}_{0 \rightarrow \infty}\right)$ was calculated according to the following formula:

$$
A U C_{0 \rightarrow \infty}=A U C_{0 \rightarrow t}+C_{p} /\left[\operatorname{Ln}(2) / t_{1 / 2}\right]
$$

The elimination half-life $\left(\mathrm{t}_{1 / 2}\right)$ was calculated as:

$$
t_{1 / 2}=\operatorname{Ln}(2) /(-b)
$$

$b$ was obtained as the slope of the linear regression of the $\log _{e}$ transformed plasma concentrations versus time in the terminal period of the plasma curve.

The pharmacokinetic calculations were performed using the computer program Kinetica $^{\mathrm{in}} 2000$.

\section{Statistical methods}

Statistical analysis was performed using the validated Kinetica ${ }^{\mathrm{Tm}}$ program. The ratios of the geometric means used to test bioequivalence were calculated from loge transformed data for $\mathrm{C}_{\max }, \mathrm{AUC}_{0 \rightarrow \mathrm{t}}$ and $\mathrm{AUC}_{0 \rightarrow \infty}$. The differences between the $\log _{\mathrm{e}}$ means and the $90 \%$ confidence interval of the difference (derived from the residual variance from the ANOVA model) were back-transformed to estimate the ratio of the two formulations and the confidence interval. $\mathrm{T}_{\max }$ was compared between formulations and fast/fed states using the Wilcoxon signed rank test and the median differences and 95\% CIs were determined using Hodges-Lehman estimates.

\section{Safety}

Evaluation of safety was based on the reported adverse events, 
Citation: Atkinson HC, Stanescu I, Beasley CPH, Salem II, et al. (2015) A Pharmacokinetic Analysis of a Novel Fixed Dose Oral Combination of Paracetamol and Ibuprofen, with Emphasis on Food Effect. J Bioequiv Availab 7: 150-154. doi:10.4172/jbb.1000230

laboratory tests, electrocardiograms, physical examination and vital signs measurements.

\section{Results}

\section{Participants}

44 subjects were screened, of which 28 were enrolled and 26 completed the crossover and were included in the pharmacokinetic analysis. The baseline demographic data are presented in Table 1.

\section{Pharmacokinetic results}

In the fasted state the pharmacokinetic parameters $\left(\mathrm{C}_{\max }, \mathrm{AUC}_{0 \rightarrow t}\right.$ and $\left.\mathrm{AUC}_{0 \rightarrow \infty}\right)$ for paracetamol and ibuprofen was similar between the fixed dose combination and mono-components. The $90 \%$ confidence intervals for the ratios of the geometric means for $\mathrm{C}_{\max }, \mathrm{AUC}_{0 \rightarrow \mathrm{t}}$, and $\mathrm{AUC}_{0 \rightarrow \infty}$ were all within the $80 \%$ to $125 \%$ bioequivalence range (Table 2).

\begin{tabular}{|c|c|}
\hline Characteristic & Study Population, $\mathbf{n = 2 8}$ \\
\hline Age, years & $28 \pm 6.51$ \\
\hline Height, cm & $172 \pm 6.79$ \\
\hline Weight, kg & $76 \pm 8.18$ \\
BMl, kg/m ${ }^{2}$ & $25.5 \pm 3.07$ \\
Smoker & $26(93)$ \\
\hline
\end{tabular}

Data presented as mean \pm SD, or $n(\%)$

Table 1: Demographic data.
In the fasted state, the median time to reach the maximum measured plasma concentration $\left(t_{\max }\right)$ was slightly faster for paracetamol and slower for ibuprofen when administered as fixed dose combination compared to monotherapy. The median $t_{\text {max }}$ for paracetamol was 38 minutes for the monotherapy and 30 minutes for the combination, whereas the median $t_{\max }$ for ibuprofen increased from 60 to 90 minutes between the mono and combination therapy.

For the fixed dose combination in the fed state, the median $t_{\text {max }}$ of paracetamol was extended by 23 minutes versus the fasted state. Conversely, the median $t_{\max }$ for ibuprofen was 37 minutes shorter in the fed state compared to the fasted state (Table 3, Figure 1). These differences were not statistically significant $(p>0.05)$.

The changes in $t_{\max }$ corresponded with a significant change in the $\mathrm{C}_{\max }$ for paracetamol as the $90 \%$ confidence interval for the ratios of the geometric means for $\mathrm{C}_{\max }$ were not within the $80-125 \%$ bioequivalence range (90\% CI: 76.37-104.47). The change in $\mathrm{C}_{\max }$ for ibuprofen was smaller and within the bioequivalence range (86.56-104.18). The changes observed in the $t_{1 / 2}$ mirrored those of the $t_{\max }$ : the half-lives of paracetamol and ibuprofen were increased and reduced, respectively, however these changes were not statistically significant.

Despite changes in the $\mathrm{C}_{\max }$, comparisons of $\mathrm{AUC}_{0 \rightarrow \mathrm{t}}$ and $\mathrm{AUC}_{0 \rightarrow \infty}$ for Maxigesic ${ }^{\mathbb{B}}$ in fed and fasted states demonstrated that the extent of absorption of both paracetamol and ibuprofen was not significantly affected by food intake. Although values for the fed state were generally

\begin{tabular}{|c|c|c|c|c|}
\hline & \multicolumn{2}{|c|}{ Paracetamol } & \multicolumn{2}{|l|}{ Ibuprofen } \\
\hline & Maxigesic $^{\circledR}$ & Monotherapy & Maxigesic $^{\circledR}$ & Monotherapy \\
\hline$C_{\max }\left(\mu g \cdot m L^{-1}\right)$ & 13.89 & 14.87 & 21.48 & 22.08 \\
\hline $\begin{array}{l}\text { Geometric mean ratio } \\
{[\%],(90 \% \mathrm{Cl})}\end{array}$ & \multicolumn{2}{|c|}{$95.77(81.62,112.37)^{b}$} & \multicolumn{2}{|c|}{$97.23(85.28,110.85)^{b}$} \\
\hline $\mathrm{AUC}_{0-\mathrm{t}}\left(\mu \mathrm{g} \cdot \mathrm{mL}^{-1} \cdot \mathrm{h}\right)$ & 38.32 & 36.51 & 80.12 & 78.56 \\
\hline $\begin{array}{l}\text { Geometric mean ratio } \\
{[\%],(90 \% \mathrm{Cl})}\end{array}$ & \multicolumn{2}{|c|}{$103.86(100.16,107.71)^{b}$} & \multicolumn{2}{|c|}{$101.18(95.72,106.97)^{\mathrm{b}}$} \\
\hline $\mathrm{AUC}_{0 \text {-inf }}\left(\mu \mathrm{g} \cdot \mathrm{mL}^{-1} \cdot \mathrm{h}\right)$ & 40.47 & 38.42 & 82.30 & 80.84 \\
\hline $\begin{array}{l}\text { Geometric mean ratio } \\
{[\%],(90 \% \mathrm{Cl})}\end{array}$ & \multicolumn{2}{|c|}{$104.08(100.15,108.15)^{b}$} & \multicolumn{2}{|c|}{$101.07(96.01,106.41)^{b}$} \\
\hline $\mathrm{T}_{\max }(\text { minutes })^{\mathrm{a}}$ & 30 & 38 & 90 & 60 \\
\hline $\mathrm{T}_{1 / 2}(\mathrm{~h})$ & 3.14 & 3.11 & 2.12 & 2.29 \\
\hline
\end{tabular}

amedian

${ }^{\mathrm{b}}$ within bioequivalence limits $(80 \%-125 \%)$

Table 2: Comparison of the geometric mean (unless stated otherwise) pharmacokinetic parameters of paracetamol and ibuprofen after administration as monotherapy or fixed-dose combination, Maxigesic ${ }^{\circledR}$ in the fasted state.

\begin{tabular}{|c|c|c|c|c|}
\hline & \multicolumn{2}{|c|}{ Paracetamol } & \multicolumn{2}{|l|}{ Ibuprofen } \\
\hline & $\begin{array}{l}\text { Maxigesic }{ }^{\circledR} \\
\text { - fed }\end{array}$ & $\begin{array}{l}\text { Maxigesic } \\
\text { - fasted }\end{array}$ & $\begin{array}{l}\text { Maxigesic }{ }^{\circledR} \\
\text { - fed }\end{array}$ & $\begin{array}{l}\text { Maxigesic } \\
\text { - fasted }\end{array}$ \\
\hline$C_{\max }\left(\mu g \cdot m L^{-1}\right)$ & 12.94 & 13.89 & 20.39 & 21.48 \\
\hline $\begin{array}{l}\text { Geometric mean ratio fed/fasted } \\
{[\%],(90 \% \mathrm{Cl})}\end{array}$ & \multicolumn{2}{|c|}{$89.32(76.37$ - 104.47) } & \multicolumn{2}{|c|}{$94.96(86.56-104.18)^{b}$} \\
\hline$A U C_{0-t}\left(\mu \mathrm{g} \cdot \mathrm{mL}^{-1} \cdot \mathrm{h}\right)$ & 38.11 & 38.32 & 70.25 & 80.12 \\
\hline $\begin{array}{l}\text { Geometric mean ratio fed/fasted } \\
{[\%],(90 \% \mathrm{Cl})}\end{array}$ & \multicolumn{2}{|c|}{$99.42(95.89-103.08)^{b}$} & \multicolumn{2}{|c|}{$88.41(85.47-91.43)^{b}$} \\
\hline$A U C_{0-\text { inf }}\left(\mu \mathrm{g} \cdot \mathrm{mL}^{-1} \cdot \mathrm{h}\right)$ & 40.80 & 40.47 & 72.20 & 82.30 \\
\hline $\begin{array}{l}\text { Geometric mean ratio fed/fasted } \\
{[\%],(90 \% \mathrm{Cl})}\end{array}$ & \multicolumn{2}{|c|}{$100.78(97.19-104.48)^{b}$} & \multicolumn{2}{|c|}{$88.40(85.80-91.07)^{b}$} \\
\hline $\mathbf{T}_{\max }(\text { minutes })^{\mathrm{a}}$ & 53 & 30 & 53 & 90 \\
\hline$T_{1 / 2}(h)$ & 3.47 & 3.14 & 2.08 & 2.12 \\
\hline
\end{tabular}

amedian

${ }^{b}$ within bioequivalence limits $(80 \%-125 \%)$

Table 3: Comparison of the geometric mean (unless stated otherwise) pharmacokinetic parameters of paracetamol and ibuprofen after administration as Maxigesic ${ }^{\circledR}$ in fed and fasted states. 


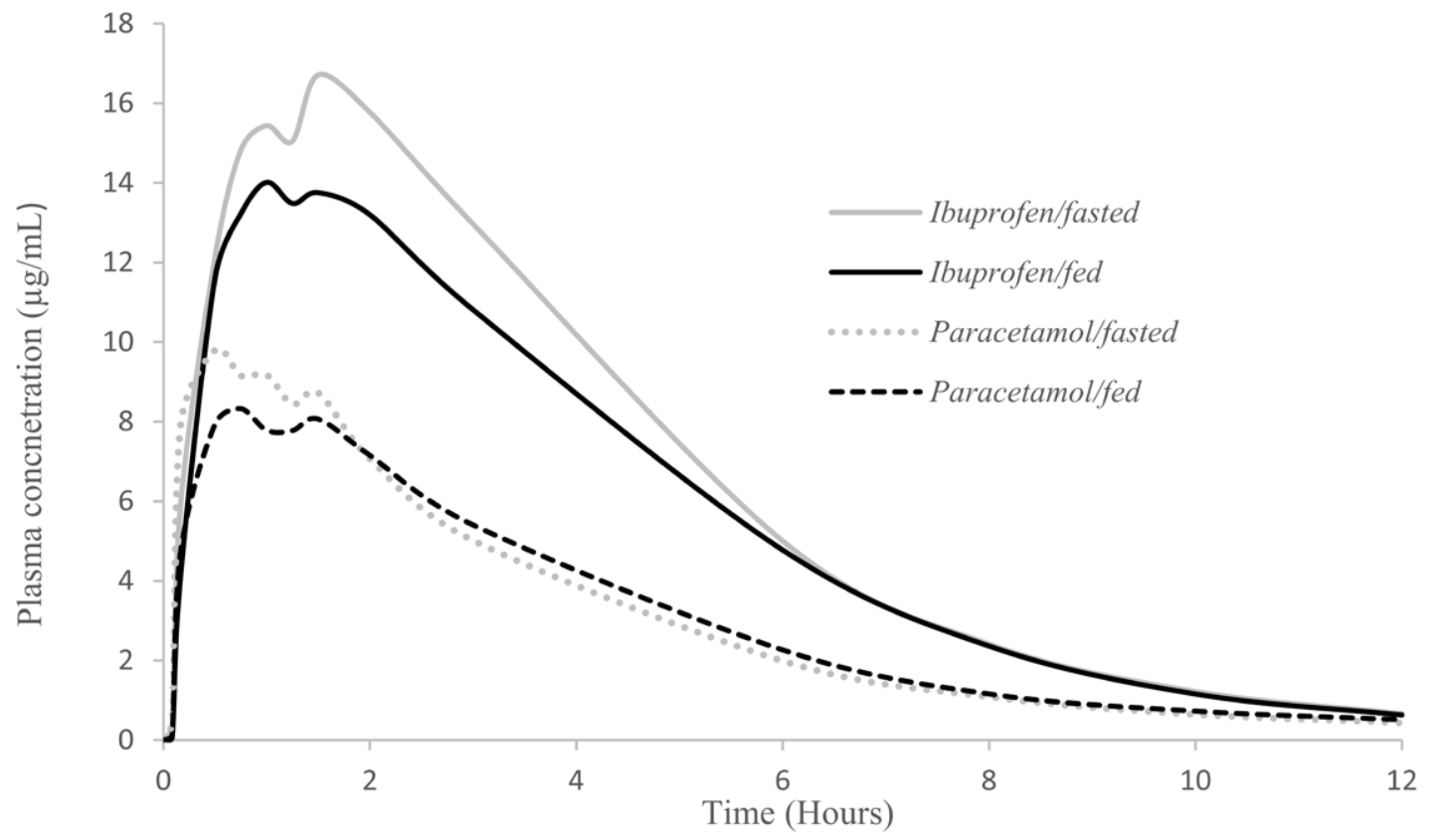

Figure 1: Mean paracetamol and ibuprofen plasma concentration versus time curves after administration of Maxigesic ${ }^{\circledR}$ in fed and fasted states

lower than in the fasted state, the $90 \%$ confidence intervals for the ratios of the geometric mean for Maxigesic ${ }^{\circledR}$ were within the $80 \%$ to $125 \%$ bioequivalence range (Table 3 ).

\section{Safety analysis}

No adverse events occurred during the entire course of this study. The study drugs were well tolerated by all participants in the study.

\section{Discussion}

The results of this study demonstrate the lack of a pharmacokinetic interaction between paracetamol and ibuprofen when administered as a novel oral fixed dose combination (Maxigesic ${ }^{\circledR}$ ) in the fasted state. Although there were differences observed in the rate of absorption after administration of mono and combination therapies, these differences were not significant and did not result in statistically significant alterations of $\mathrm{C}_{\max }, \mathrm{AUC}_{0 \rightarrow \mathrm{t}}$ or $\mathrm{AUC}_{0 \rightarrow \infty}$ as the $90 \%$ CIs for the ratio of the geometric means were within the $80 \%-125 \%$ accepted bioequivalence range.

These results are in agreement with the literature on the pharmacokinetics of concurrent administration of ibuprofen and paracetamol $[2,3]$. In the fasted state there were no significant differences in the mean $\mathrm{C}_{\max }$ and $\mathrm{AUC}$ of ibuprofen or paracetamol when the ingredients are administered alone or in a fixed dose combination of ibuprofen $400 \mathrm{mg}$ and paracetamol $650 \mathrm{mg}$ (henceforth 'FDC 400/650') [2], or a fixed dose combination of ibuprofen $400 \mathrm{mg}$ and paracetamol $1000 \mathrm{mg}$ (henceforth 'FDC 400/1000') [3].

In the present study, the median $\mathrm{t}_{\max }$ of paracetamol was 8 minutes shorter for Maxigesic ${ }^{\circledR}$, compared to the monotherapy in the fasted state. Similarly, the FDC 400/1000 and FDC 400/650 result in paracetamol $\mathrm{t}_{\max }$ values that are shorter by $10(\mathrm{p}<0.05)$ and $6(\mathrm{p}>0.05)$ minutes, respectively $[2,3]$ (Table 4 ).

While there is a large body of literature demonstrating the pharmacokinetics of paracetamol and ibuprofen monotherapies, the literature concerning fixed dose combinations of such ingredients is comparatively scant. A single food effect study was identified concerning the FDC 400/1000 [3] (the food effect has not been evaluated for the FDC 400/650). The $90 \%$ CIs of $\mathrm{AUC}_{0 \rightarrow \mathrm{t}}$ and $\mathrm{AUC}_{0 \rightarrow \infty}$ between fed and fasted states for both ibuprofen and paracetamol in the FDC $400 / 1000$ are within the accepted $80-125 \%$ bioequivalence range. However, the $90 \%$ CIs for both paracetamol and ibuprofen $\mathrm{C}_{\max }$ show a lack of bioequivalence for both ingredients $(53.4 \%-69.5 \%$, $70.3 \%-83.1 \%$, respectively). The present study demonstrated that the $\mathrm{AUC}_{0 \rightarrow \mathrm{t}}$ and $\mathrm{AUC}_{0 \rightarrow \infty}$ of both ingredients in Maxigesic ${ }^{\circledR}$ are not altered in the fed state. These data suggest that a smaller dose of ibuprofen (300 $\mathrm{mg}$ in Maxigesic ${ }^{\mathbb{B}}$ vs. $400 \mathrm{mg}$ in the FDC 400/1000) results in a smaller attenuation of absorption of both ingredients in the fed state compared to the fasted state.

Administration of the FDC $400 / 1000$ after a meal results in a significant increase in median paracetamol $\mathrm{t}_{\text {max }}$ of 60 minutes $(\mathrm{p}<0.001$, 95\% CI: 30-80) to 90 minutes, and non-significant extension of median ibuprofen $\mathrm{t}_{\max }$ of 45 minutes ( $\mathrm{p}>0.05,95 \%$ CI: $0-45$ ) to 120 minutes and unlike the present study, the two ingredients in this combination do not reach maximal concentration at the same time (Table 4). As expected, the lower dose of ibuprofen in Maxigesic ${ }^{\circledR}(300 \mathrm{mg})$ resulted in a shorter $t_{\max }$ in the fed state than the FDC 400/1000 (53 and 120 minutes in the fed state, respectively), however, despite the dose of paracetamol being the same, the $t_{\max }$ of paracetamol was 53 minutes for Maxigesic $^{\circledR}$ and 90 minutes for the FDC 400/1000. Comparatively, the food effect is less pronounced in Maxigesic ${ }^{\circledR}$ and the active ingredients reach maximal plasma concentration earlier than in a fixed dose combination of ibuprofen $400 \mathrm{mg}$ and paracetamol $1000 \mathrm{mg}$ (FDC $400 / 1000)$, and at the same time in the fed state (53 minutes). The shorter and coordinated time to $\mathrm{C}_{\text {max }}$ for both ingredients in Maxigesic ${ }^{\mathbb{R}}$ may be clinically relevant, resulting in a greater analgesic effect with an earlier time of onset. Furthermore, Maxigesic ${ }^{\circledR}$ at normal daily dosages 
Citation: Atkinson HC, Stanescu I, Beasley CPH, Salem II, et al. (2015) A Pharmacokinetic Analysis of a Novel Fixed Dose Oral Combination of Paracetamol and Ibuprofen, with Emphasis on Food Effect. J Bioequiv Availab 7: 150-154. doi:10.4172/jbb.1000230

\begin{tabular}{|c|c|c|c|c|c|}
\hline \multirow{2}{*}{$T_{\max }$ (minutes) } & \multirow{2}{*}{$n$} & \multicolumn{2}{|l|}{ Paracetamol } & \multicolumn{2}{|l|}{ Ibuprofen } \\
\hline & & Combination - fasted & Monotherapy -fasted & Combination - fasted & Monotherapy -fasted \\
\hline FDC $300 / 1000\left(\right.$ Maxigesic $\left.^{\circledR}\right)$ & 26 & 30 & 38 & 90 & 60 \\
\hline Median difference $(95 \% \mathrm{Cl})$ & & \multicolumn{2}{|l|}{$-6.4(-22,15)$} & \multicolumn{2}{|l|}{$22.5(-22,60)$} \\
\hline $\begin{array}{l}\text { FDC400/650 } \\
\text { (mean [SD]) }\end{array}$ & 20 & $48(24)$ & $54(30)$ & $84(42)$ & $84(42)$ \\
\hline FDC $400 / 1000$ & 25 & 30 & 40 & 75 & 75 \\
\hline \multirow[t]{2}{*}{ Median difference $(95 \% \mathrm{Cl})$} & & \multicolumn{2}{|l|}{$-15(-30,0)$} & \multicolumn{2}{|l|}{$7.5(-15,37.5)$} \\
\hline & & $\begin{array}{l}\text { Combination } \\
\text { - fed }\end{array}$ & Combination - fasted & $\begin{array}{l}\text { Combination } \\
\text { - fed }\end{array}$ & Combination - fasted \\
\hline FDC $300 / 1000$ (Maxigesic $^{\circledR}$ ) & 26 & 53 & 30 & 53 & 90 \\
\hline \multicolumn{2}{|l|}{ Median difference $(95 \% \mathrm{Cl})$} & \multicolumn{2}{|l|}{$22.5(0,60)$} & \multicolumn{2}{|l|}{$-22.5(-45,0)$} \\
\hline FDC $400 / 1000$ & 25 & 90 & 30 & 120 & 75 \\
\hline \multicolumn{2}{|l|}{ Median difference $(95 \% \mathrm{Cl})$} & \multicolumn{2}{|l|}{$55(30,80)$} & \multicolumn{2}{|l|}{$25(0,45)$} \\
\hline
\end{tabular}

ano fast-fed comparison has been published for FDC 400/650

Table 4: Tmax for ibuprofen and paracetamol after single dose administration of fixed dose combinations: 300/1000 (Maxigesic ${ }^{\circledR}$ ), $400 / 650$ and $400 / 1000$.

provides a lower cumulative dose of ibuprofen than the FDC 400/1000 and thereby offers a potentially lower risk of class-related adverse events such as nausea and gastrointestinal ulceration.

\section{Conclusion}

The concomitant administration of ibuprofen and paracetamol in a fixed dose combination (Maxigesic ${ }^{\mathbb{R}}$ ) does not alter the pharmacokinetic profiles of either drug in the fasted state and there was no effect of food on the absorption from the fixed dose combination. These results are largely in accord with the literature on the pharmacokinetics of monotherapy or combination doses of ibuprofen and paracetamol.

\section{Acknowledgements}

We thank the staff at IPRC for the administration of the study protocol and data collection. We are grateful to the volunteers who participated in this study. This study was funded by AFT Pharmaceuticals Ltd, Auckland, NZ.

\section{Author Contributions}

$\mathrm{H}$ Atkinson supervised the project and was involved in the design of the study, development of the protocol, interpretation of the data and writing of the manuscript. I Stanescu was involved in the study design, protocol development, and writing of the manuscript. C Beasley contributed to the statistical data interpretation and assisted in drafting the manuscript. I Salem performed the plasma drug assays data analysis and interpretation and reviewed the manuscript. C Frampton critically reviewed the manuscript.

\section{Conflicts of Interest}

$\mathrm{H}$ Atkinson is a shareholder and Managing Director of AFT Pharmaceuticals, and I Stanescu and C Beasley are Employees of AFT Pharmaceuticals Ltd. I Salem is an employee of IPRC. C Frampton provides consultancy services to AFT Pharmaceuticals Ltd.

\section{References}

1. Merry AF, Gibbs RD, Edwards J, Ting GS, Frampton C et al. (2010) Combined acetaminophen and ibuprofen for pain relief after oral surgery in adults: a randomized controlled trial. $\mathrm{Br} \mathrm{J}$ Anaesth 104: 80-88.

2. Wright CE, Antal EJ, Gillespie WR, Albert KS (1983) Ibuprofen and acetaminophen kinetics when taken concurrently. Clin Pharmacol Ther 34: 707-710

3. Tanner T, Aspley S, Munn A, Thomas T (2010) The pharmacokinetic profile of a novel fixed-dose combination tablet of ibuprofen and paracetamol. BMC Pharmacol Toxicol 10: 10.

4. Hattori N, Hasegawa K, Sakamoto T (2012) Pharmacokinetics and effect of food after oral administration of prolonged-release tablets of ropinirole hydrochloride in Japanese patients with Parkinson's disease. J Clin Pharm Ther 37: 571-577.

5. Choi HY, Noh YH, Kim YH, Kim MJ, Lee SH, Kim JA, et al. (2014) Effects of food on the pharmacokinetics of gemigliptin/metformin sustained-release
$50 / 1,000 \mathrm{mg}(25 / 500 \mathrm{mg} \times 2$ tablets) fixeddose combination tablet in healthy male volunteers. J Clin Pharmacol 52: 381-391.

6. Damle B, Duczynski G, Jeffers BW, Crownover P, Coupe A, LaBadie RR (2014) Pharmacokinetics of a novel orodispersible tablet of sildenafil in healthy subjects. Clin Ther 36: 236-244.

7. Liu D, Jiang J, Zhang L, Tan F, Wang Y, Zhang D, et al. (2014) Clinical pharmacokinetics of Icotinib, an anti-cancer drug: evaluation of dose proportionality, food effect, and tolerability in healthy subjects. Cancer Chemother Pharmacol 73: 721-727.

8. Lv C, Wei C, Wang X, Yao H, Li R, Wang B, et al. (2014) The influence of food on the pharmacokinetics of amlodipine and losartan after single-dose of its compound tablets in healthy Chinese subjects. Drug Res 64: 229-235.

9. Shaik MN, LaBadie RR, Rudin D, Levin WJ (2014) Evaluation of the effect of food and ketoconazole on the pharmacokinetics of the smoothened inhibitor PF-04449913 in healthy volunteers. Cancer Chemother Pharmacol 74: 411418.

10. Singh BN (2012) Effects of Food on Clinical Pharmacokinetics. Clin Pharmacokinet 37: 213-255.

11. Siemon D, de Vries JX, Stötzer F, Walter-Sack I, Dietl R (1997) Fasting and postprandial disposition of $R(-)$-and $S(+)$-ibuprofen following oral administration of racemic drug in healthy individuals. Eur J Med Res 2: 215-219.

12. Pargal A, Kelkar MG, Nayak PJ (1996) The Effect of Food on the Bioavailability of Ibuprofen and Flurbiprofen from Sustained Release Formulations. Biopharm Drug Dispos 17: 511-519.

13. Borin MT, Khare S, Beihn RM, Jay M (1990) The Effect of Food on Gastrointestinal (GI) Transit of Sustained-Release Ibuprofen Tablets as Evaluated by Gamma Scintigraphy. Pharm Res 7: 304-307.

14. Kapil R, Nolting A, Roy P, Fiske W, Benedek I, Abramowitz W (2004) Pharmacokinetic properties of combination oxycodone plus racemic ibuprofen Two randomized, open-label, crossover studies in healthy adult volunteers Clin Ther 26: 2015-2025.

15. Divoll M, Greenblatt DJ, Ameer B, Abernethy DR (1982) Effect of Food on Acetaminophen Absorption in Young and Elderly Subjects. J Clin Pharmacol 22: $571-576$

16. Stillings M, Havlik I, Chetty M, Clinton C, Schall R, Moodley I, et al. (2000) Comparison of the Pharmacokinetic Profiles of Soluble Aspirin and Solid Paracetamol Tablets in Fed and Fasted Volunteers. Curr Med Res Opin 16: $115-124$.

17. Smith S, Collaku A, Heaslip L, Yue Y, Starkey Y-Y, Clarke G, et al. (2012) A new rapidly absorbed paediatric paracetamol suspension. A six-way crossover pharmacokinetic study comparing the rate and extent of paracetamol absorption from a new paracetamol suspension with two marketed paediatric formulations. Drug Dev Ind Pharm 38: 372-379.

18. Armstrong J, Challenor VF, Macklin BS, Renwick AG, Waller DG (1997) The influence of two types of meal on the pharmacokinetics of a modified-release formulation of nifedipine (Adalat Retard). Eur J Clin Pharmacol 53: 141-143. 